\title{
Supporting Information For: Correlating Broadband Photoluminescence with Structural Dynamics in Layered Hybrid Halide Perovskites
}

Alexandra A. Koegel, ${ }^{\dagger}$ Eve M. Mozur, ${ }^{\dagger}$ Iain W. H. Oswald,${ }^{\dagger}$ Niina H. Jalarvo, ${ }^{\ddagger}$ Timothy R. Prisk, ${ }^{\mathrm{II}}, \|$ Madhusudan Tyagi, ${ }^{\mathrm{II}}{ }^{\S}$ and James R. Neilson*,†

$\dagger$ Colorado State University, Department of Chemistry, Fort Collins, Colorado 80523-1872, United States

$\$$ Neutron Scattering Division, Oak Ridge National Laboratory, Oak Ridge, Tennessee 37831, United States

IINIST Center for Neutron Research National Institute of Standards and Technology, Gaithersburg, Maryland 20899, United States

$\S$ Department of Materials Science and Engineering, University of Maryland, College Park, Maryland 20742, United States

||Present address: Division of Chemistry and Chemical Engineering, California Institute of Technology, Pasadena, California 91125, United States.

E-mail: james.neilson@colostate.edu 


\section{Supplemental Information}

\section{Preliminary Characterization}

For the compounds synthesized in this study, PXRD data were modeled with space groups of $P b c a$ for $(n \mathrm{BA})_{2} \mathrm{PbBr}_{4}, P 2_{1} / c$ for (ODA) $\mathrm{PbBr}_{4}$, and $P 2_{1} / c$ for $(\mathrm{GABA})_{2} \mathrm{PbBr}_{4}$ using previously reported structures ${ }^{1}$ with the Rietveld method implemented in TOPAS v6 (Bruker AXS). The room temperature photoluminescence spectroscopy reveals emission peaked near $415 \mathrm{~nm}$ (Figure S2), in agreement with a previous study. 1

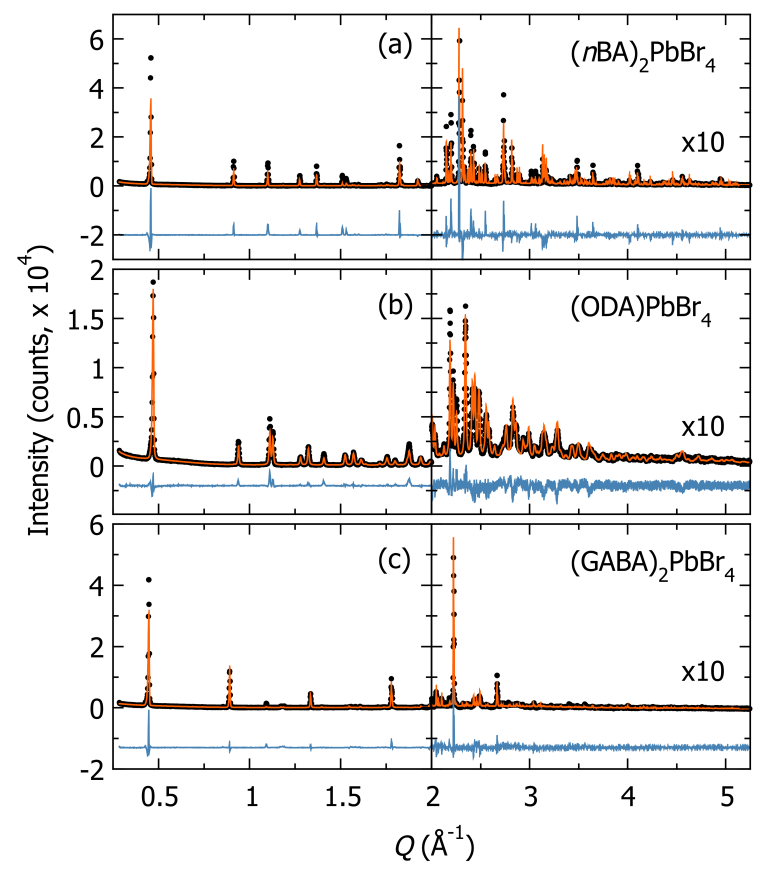

Figure S1: Powder X-ray diffraction of (a) $(n \mathrm{BA})_{2} \mathrm{PbBr}_{4}$, (b) (ODA) $\mathrm{PbBr}_{4}$, and (c) $(\mathrm{GABA})_{2} \mathrm{PbBr}_{4}$. Black circles, orange line, and blue line represent the data, Rietveld refinement, and difference curve, respectively. 


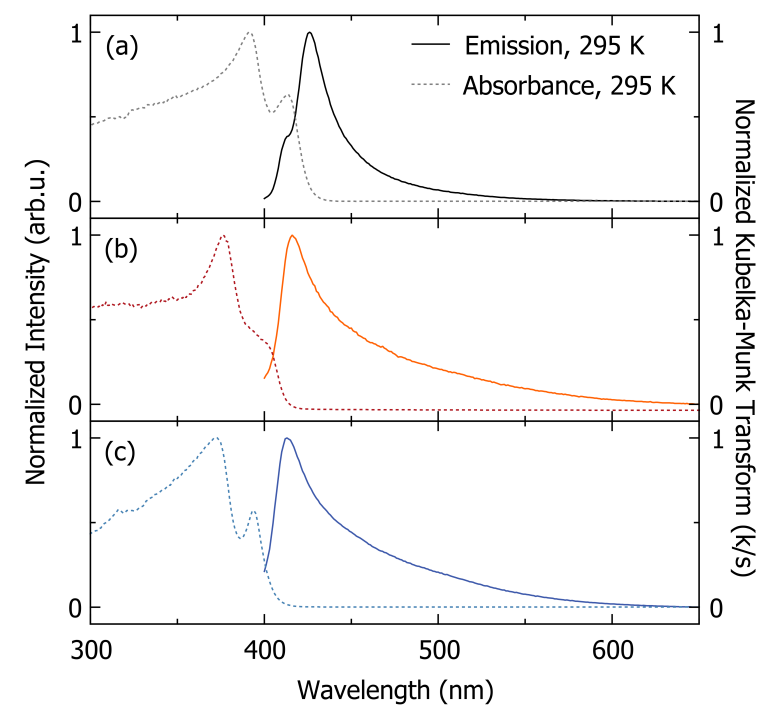

Figure S2: Ambient temperature (295 K) normalized photoluminescence intensity with excitation at $375 \mathrm{~nm}$ (solid lines, left axis) showing narrow emission centered at $415 \mathrm{~nm}$ and Kubelka-Munk transform (k/s) (dotted lines, right axis) of (a) $(n \mathrm{BA})_{2} \mathrm{PbBr}_{4}$ (black), (b) (ODA) $\mathrm{PbBr}_{4}$ (red), and (c) $(\mathrm{GABA})_{2} \mathrm{PbBr}_{4}$ (blue).

\section{Thermal activation of motion}

Activation energies vary by molecule and motion occurring within $(n \mathrm{BA})_{2} \mathrm{PbBr}_{4},(\mathrm{ODA}) \mathrm{PbBr}_{4}$, and $(\mathrm{GABA})_{2} \mathrm{PbBr}_{4}$. The organic cation dynamics do not follow classically Arrhenius behavior as seen in Figure $\mathrm{S} 3$. The data presented as $\ln (\tau)$ v. $T^{-1}$ are not linear and there are multiple relaxation times observed at $T \geq 275 \mathrm{~K}$ for $(n \mathrm{BA})_{2} \mathrm{PbBr}_{4}$ and $T \geq 275 \mathrm{~K}$ for (ODA) $\mathrm{PbBr}_{4}$. 

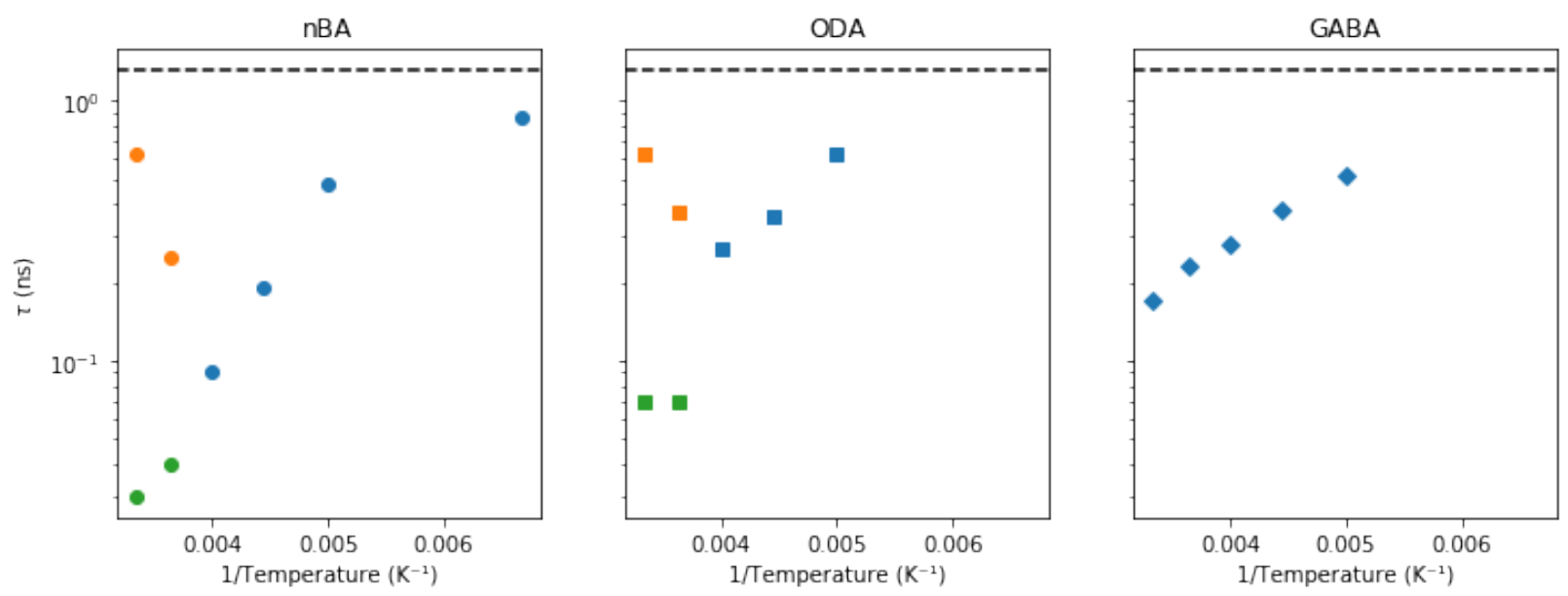

Figure S3: $\ln (\tau)$ vs. $T^{-1}$ (left) $(n \mathrm{BA})_{2} \mathrm{PbBr}_{4}$, (middle) (ODA) $\mathrm{PbBr}_{4}$, and (right) $(\mathrm{GABA})_{2} \mathrm{PbBr}_{4}$ calculated from QENS data collected from $150 \mathrm{~K}$ to $300 \mathrm{~K}$. Blue symbols indicate a singular Lorentzian. Orange and green symbols indicate two separate Lorentzian functions used to model the QENS data.

\section{Quasi-elastic scattering from $(n \mathrm{BA})_{2} \mathrm{PbBr}_{4},(\mathrm{ODA}) \mathrm{PbBr}_{4}$, and $\left(\mathrm{GABA}_{2} \mathrm{PbBr}_{4}\right.$}

There is no QENS observed at liquid nitrogen temperatures (Figure S4). This lack of resolvable QENS is due to the speed of the organic cation motions occurring within the broad elastic line at low temperatures, making quasi-elastic interactions unobservable within the resolution of the instrument. At temperatures $<200 \mathrm{~K}$ only the motions of $n \mathrm{BA}$ are observable and have a full width at half maximum (FWHM) of roughly $1.5 \mu \mathrm{eV}$ (Figures S5 and S6). 


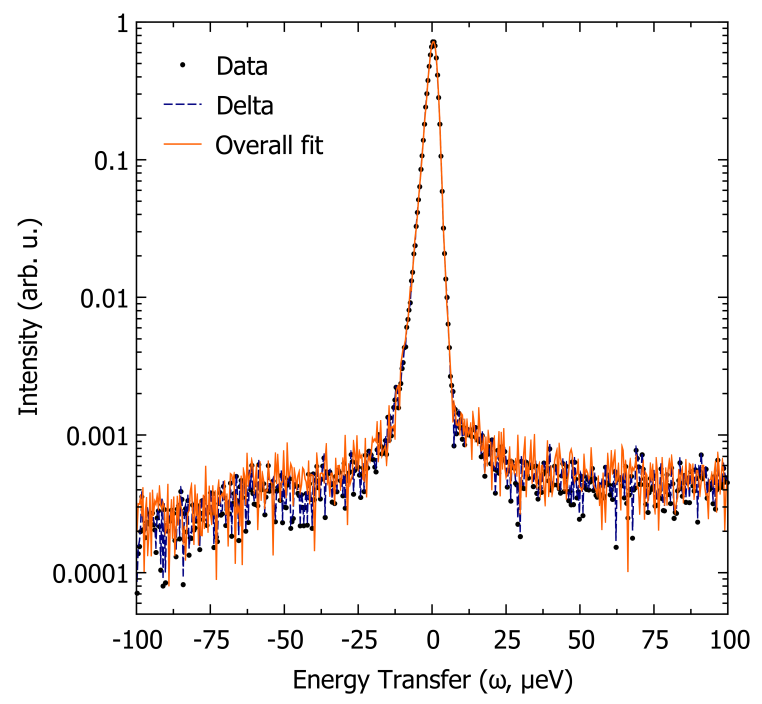

Figure S4: Representative QENS spectra of $(\mathrm{GABA})_{2} \mathrm{PbBr}_{4}$ at $T=77 \mathrm{~K}$ for $Q=1.1 \AA^{-1}$ collected at BASIS. The spectra are shown as black circles, with the overall fit shown as a solid red line. The data are modeled with a $\delta$-function convolved with an instrument resolution function collected for each sample at $T=20 \mathrm{~K}$ to account for elastic scattering, shown here as a blue dashed line. There are no quasi-elastic interactions present.

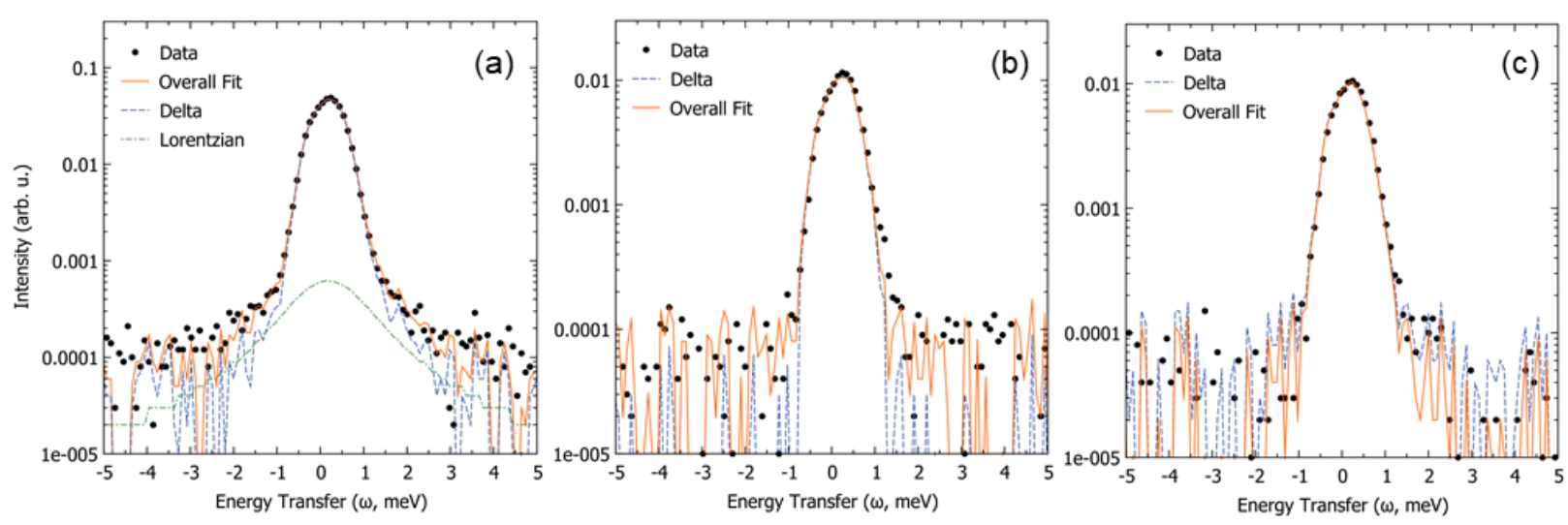

Figure S5: Quasi-elastic neutron scattering at $150 \mathrm{~K}$ for (a) $(n \mathrm{BA})_{2} \mathrm{PbBr}_{4}$ (b) (ODA) $\mathrm{PbBr}_{4}$, and (c) $(\mathrm{GABA})_{2} \mathrm{PbBr}_{4}$ collected at HFBS. The representative spectra are shown as black circles, with the overall fit shown as a solid red line. The data are modeled with a $\delta$-function convolved with an instrument resolution function collected for each sample with a vanadium standard to account for elastic scattering, shown here as a blue dashed line. The broadening from quasi-elastic scattering is fit with a Lorentzian, shown as a green dash-dotted lines only in (a). Note the log scale on the y-axis. 


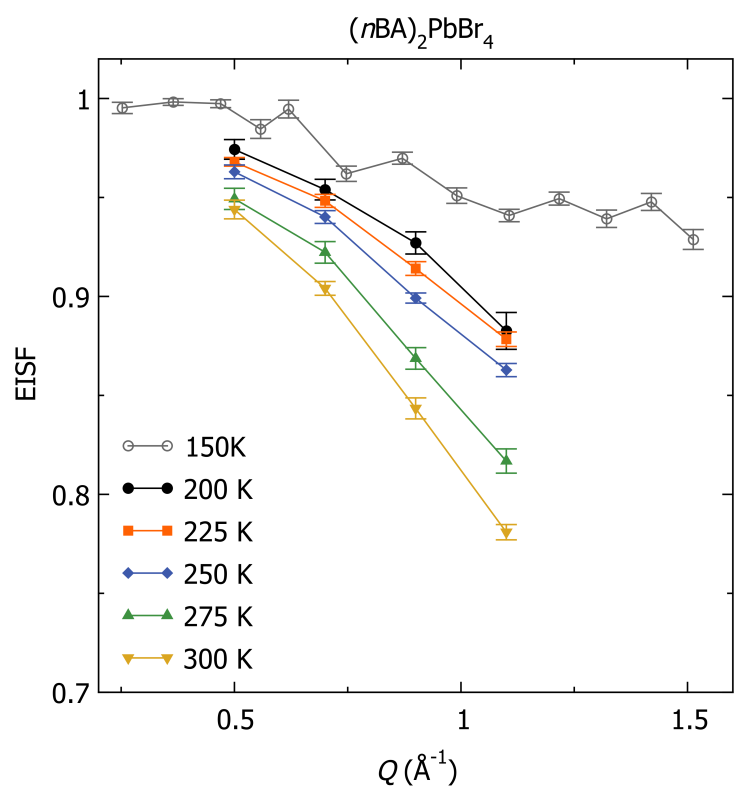

Figure S6: Elastic incoherent structure factor of $(n \mathrm{BA})_{2} \mathrm{PbBr}_{4}$ at $150 \mathrm{~K}$ to $300 \mathrm{~K}$ extracted from quasi-elastic neutron scattering data from HFBS (150 K) and BASIS (200 K to $300 \mathrm{~K}$ ). Error bars represent one standard deviation.

\section{Rotational mode analysis of $(n \mathrm{BA})_{2} \mathrm{PbBr}_{4},(\mathrm{ODA}) \mathrm{PbBr}_{4}$, and $\left(\mathrm{GABA}_{2} \mathrm{PbBr}_{4}\right.$}

Figures $\mathrm{S} 8$ and $\mathrm{S} 9$ are the molecules with either a total model (Figure $\mathrm{S} 8$ ) or $C_{3}$ model (Figure S9) applied to the data at temperatures $200 \mathrm{~K}$ to $300 \mathrm{~K}$. Figure S8 shows the full model for $(n \mathrm{BA})_{2} \mathrm{PbBr}_{4}$ that includes $C_{2} \otimes C_{3}$ of the $-\mathrm{NH}_{3}$ headgroup (a $C_{2}$ of the $-\mathrm{CH}_{2}$ adjacent to the $-\mathrm{NH}_{3}$ terminal group with a $C_{3}$ of the ammonium headgroup), a $\mathrm{C}_{3}$ of the methyl group, and librations of the aliphatic hydrogens applied to the data at all temperatures, as depicted in Figure S7. The model describes the data at $300 \mathrm{~K}$ and does not describe the data for $T \leq 275 \mathrm{~K}$. When the model is reduced to describe only the $C_{3}$ motions of the ammonium headgroup and methyl group, the model fits the data at $275 \mathrm{~K}$ (Figure S9). All other data has an EISF representative of a more static molecule than a $C_{3}$ model implies. Therefore, fractional motion was applied to fit data for $T<275 \mathrm{~K}$. The best fit parameters for fractional $C_{3}$ motion are tabulated in Table $\mathrm{S} 2$ and plotted in Figure $\mathrm{S} 10$.

Figure $\mathrm{S8p}$ shows the results from fitting the full dynamic model for ODA in (ODA) $\mathrm{PbBr}_{4}$ that includes $C_{2} \otimes C_{3}$ of the $-\mathrm{CH}_{2} \mathrm{NH}_{3}$ headgroup in conjunction with a $C_{2}$ of the $-\mathrm{CH}_{2}$ adjacent 
to the $-\mathrm{NH}_{3}$ terminal group and librations of the aliphatic hydrogens applied to the data at all temperatures depicted in Figure $\mathrm{S7}$. This model only describes the data for $T \geq 275 \mathrm{~K}$. However, when the model is changed to only describe the $C_{3}$ motion of the ammonium head groups, it is a good fit to data $T=250 \mathrm{~K}$, whereas the EISFs at $T \leq 225 \mathrm{~K}$ describe a more static molecule than the model. Therefore, fractional motion was applied to fit the low temperature data. The best fit parameters for fractional $C_{3}$ motion are tabulated in Table $\mathrm{S} 2$ and plotted in Figure $\mathrm{S} 10$.

Figure $\mathrm{S} 8 \mathrm{c}$ shows the full model for $(\mathrm{GABA})_{2} \mathrm{PbBr}_{4}$ that includes $C_{2} \otimes C_{3}$ of the $-\mathrm{CH}_{2} \mathrm{NH}_{3}$ headgroup in conjunction with a $C_{2}$ of the $-\mathrm{CH}_{2}$ adjacent to the $-\mathrm{NH}_{3}$ terminal group and $-\mathrm{COOH}$ group, and librations of the aliphatic hydrogens applied to the data at all temperatures depicted in Figure S7. This model does not describe any of the data. When the motion is reduced to a model describing only $C_{3}$ motions, the data at $T=300 \mathrm{~K}$ fits well; however, none of the other temperatures are described. Therefore, a fractional $C_{3}$ model was applied. The best fit parameters for fractional $C_{3}$ motion are tabulated in Table $\mathrm{S} 2$ and plotted in Figure $\mathrm{S} 10$.

Relaxation times of the principle hopping motions of the cations described above are listed in Table $\mathrm{S} 1$ and shown in Figure $\mathrm{S11}$. Extrapolated relaxation times were calculated with a least squares polynomial fit in Python. The $n \mathrm{BA}$ cations have the shortest measured relaxation times at all temperatures except $77 \mathrm{~K}$, where GABA has the shortest extrapolated relaxation time.

Table S1: Relaxation times of hydrogen motions $(\tau, \mathrm{ns})$ extracted from QENS spectra. Error bars represent one standard deviation.

\begin{tabular}{|c|c|c|c|}
\hline & \multicolumn{3}{|c|}{ Relaxation times $^{\dagger}(\mathrm{ns})$} \\
\hline Temp (K) & $n \mathrm{BA}$ & ODA & GABA \\
\hline 77 & $5.35^{*}$ & $4.59^{*}$ & $1.94^{*}$ \\
150 & $0.86 \pm 0.1$ & $1.36^{*}$ & $0.876^{*}$ \\
200 & $0.48 \pm 0.02$ & $0.62 \pm 0.009$ & $0.52 \pm 0.05$ \\
225 & $0.19 \pm 0.004$ & $0.36 \pm 0.009$ & $0.38 \pm 0.02$ \\
250 & $0.09 \pm 0.002$ & $0.27 \pm 0.004$ & $0.28 \pm 0.01$ \\
275 & $0.25 \pm 0.07,0.04 \pm 0.005$ & $0.37 \pm 0.02,0.07 \pm 0.003$ & $0.23 \pm 0.005$ \\
300 & $0.62 \pm 0.27,0.03 \pm 0.0008$ & $0.62 \pm 0.07,0.07 \pm 0.0007$ & $0.17 \pm 0.005$ \\
\hline
\end{tabular}

* Estimated extrapolated relaxation time values. Values were extrapolated assuming $\ln (\tau)=$ $A T+B$, fitting $A$ and $B$ across the temperatures for which a single tau is recorded. (Figure S11). Uncertainty calculated for $n \mathrm{BA}$ at $150 \mathrm{~K}$ used error propagation of one $Q$-group from DAVE. 
Table S2: Best fit parameters for hydrogens undergoing fractional $C_{3}$ motion in $(n \mathrm{BA})_{2} \mathrm{PbBr}_{4}$, $(\mathrm{ODA}) \mathrm{PbBr}_{4}$, and $(\mathrm{GABA})_{2} \mathrm{PbBr}_{4}$. Errors were estimated from taking the square root of the diagonals of the covariance matrix.

\begin{tabular}{|c|c|c|c|}
\hline \multicolumn{5}{|c|}{$C_{3}$ Fraction } \\
\hline Temperature $(\mathrm{K})$ & $n \mathrm{BA}$ & ODA & GABA \\
\hline 200 & $0.60(2)$ & $0.6000(6)$ & $0.2000(3)$ \\
225 & $0.70(1)$ & $0.9000(3)$ & $0.4000(2)$ \\
250 & $0.80(1)$ & - & $0.53000(4)$ \\
275 & - & - & $0.7000(8)$ \\
300 & - & - & $0.9000(7)$ \\
\hline
\end{tabular}
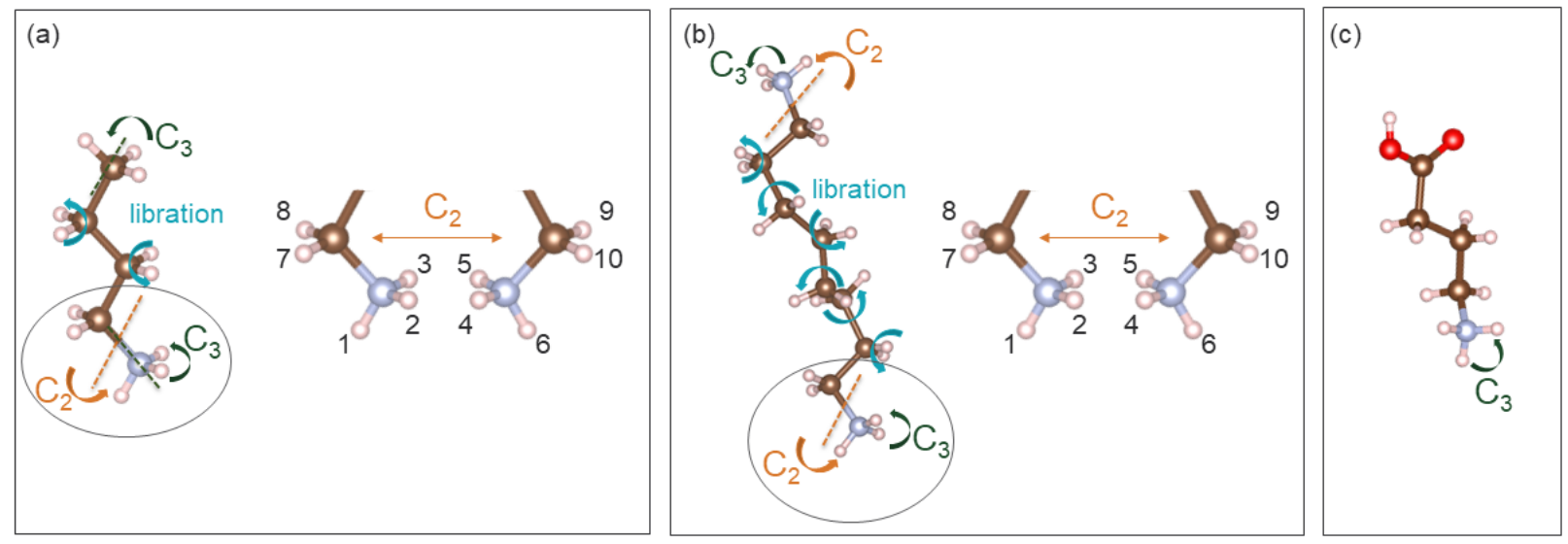

Figure S7: Visualization of rotational motions simulated in jump models in (a) $(n \mathrm{BA})_{2} \mathrm{PbBr}_{4}$, (b) (ODA) $\mathrm{PbBr}_{4}$, and (c) (GABA) ${ }_{2} \mathrm{PbBr}_{4}$ at room temperature. Green arrows indicate $C_{3}$ rotations of the $-\mathrm{NH}_{3}$ and $\mathrm{CH}_{3}$ groups. Orange arrows indicate a $C_{2}$ rotation of the $-\mathrm{CH}_{2} \mathrm{NH}_{3}$ head group about a predetermined angle as indicated in the cartoon on the left. ${ }^{2}$ Blue arrows indicate librations of the $-\mathrm{CH}_{2}$ chain. 

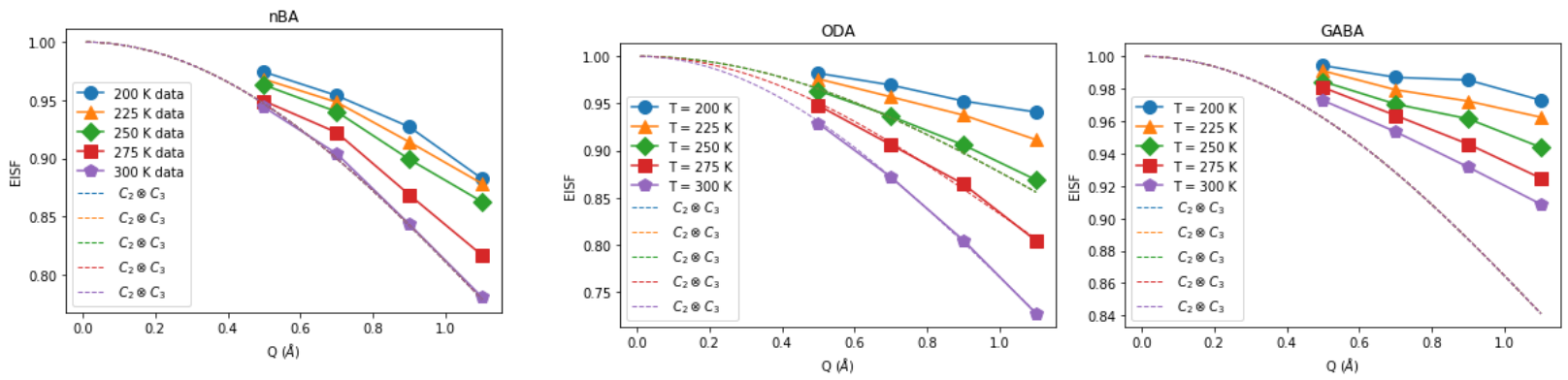

Figure S8: Elastic incoherent structure factor of (left) $(n \mathrm{BA})_{2} \mathrm{PbBr}_{4}$, (middle) (ODA) $\mathrm{PbBr}_{4}$, and (right) (GABA) $)_{2} \mathrm{PbBr}_{4}$ at $200 \mathrm{~K}$ to $300 \mathrm{~K}$ extracted from QENS data at BASIS fit with a jump model labeled as $C_{2} \otimes C_{3}$ that, depending on the molecule, includes: $C_{2} \otimes C_{3}$ of the $-\mathrm{CH}_{2} \mathrm{NH}_{3}$ headgroup, $C_{3}$ of the ammonium and methyl groups, $C_{2}$ of the $-\mathrm{CH}_{2} \mathrm{CH}_{3}$ headgroup and $-\mathrm{COOH}$, and librations of the $\mathrm{CH}_{2}$ backbone, as described above. The full model does not describe all motions at all temperatures for the three molecules studied. The models for temperatures in (a) $T$ $\leq 300 \mathrm{~K}$, (b) $T \leq 275 \mathrm{~K}$, and (c) $T \leq 300 \mathrm{~K}$ are overlapping.
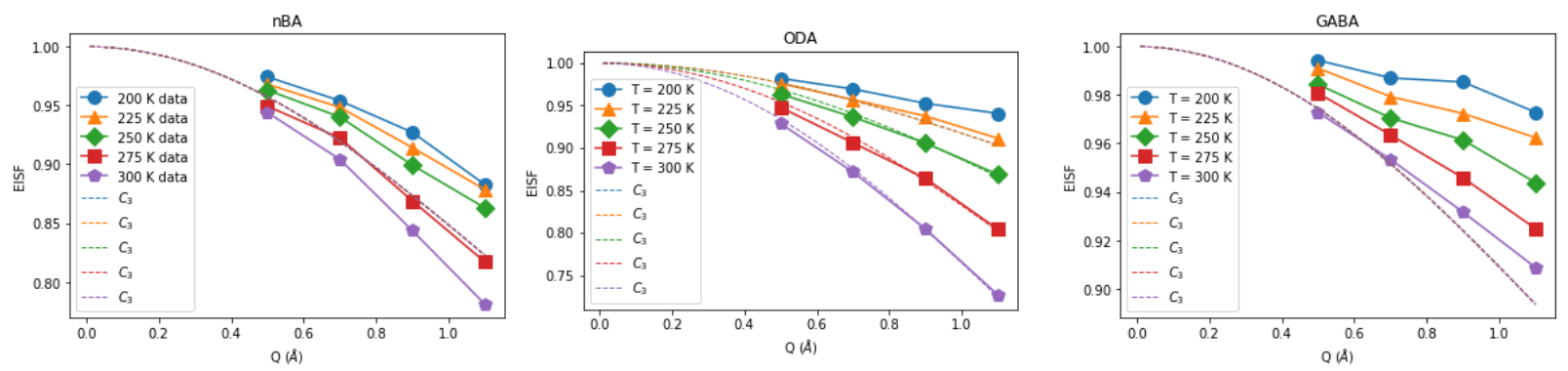

Figure S9: Elastic incoherent structure factor of (left) $(n \mathrm{BA})_{2} \mathrm{PbBr}_{4}$, (middle) (ODA) $\mathrm{PbBr}_{4}$, and (right) $(\mathrm{GABA})_{2} \mathrm{PbBr}_{4}$ at $200 \mathrm{~K}$ to $300 \mathrm{~K}$ extracted from QENS data at BASIS modeled by a jump model describing the $C_{3}$ rotations of the ammonium headgroup, as described in the narrative. This model does not describe all motions at all temperatures for the three molecules studied. The models for $T \leq 300 \mathrm{~K}$, (b) $T \leq 250 \mathrm{~K}$, and (c) $T \leq 300 \mathrm{~K}$ are overlapping 


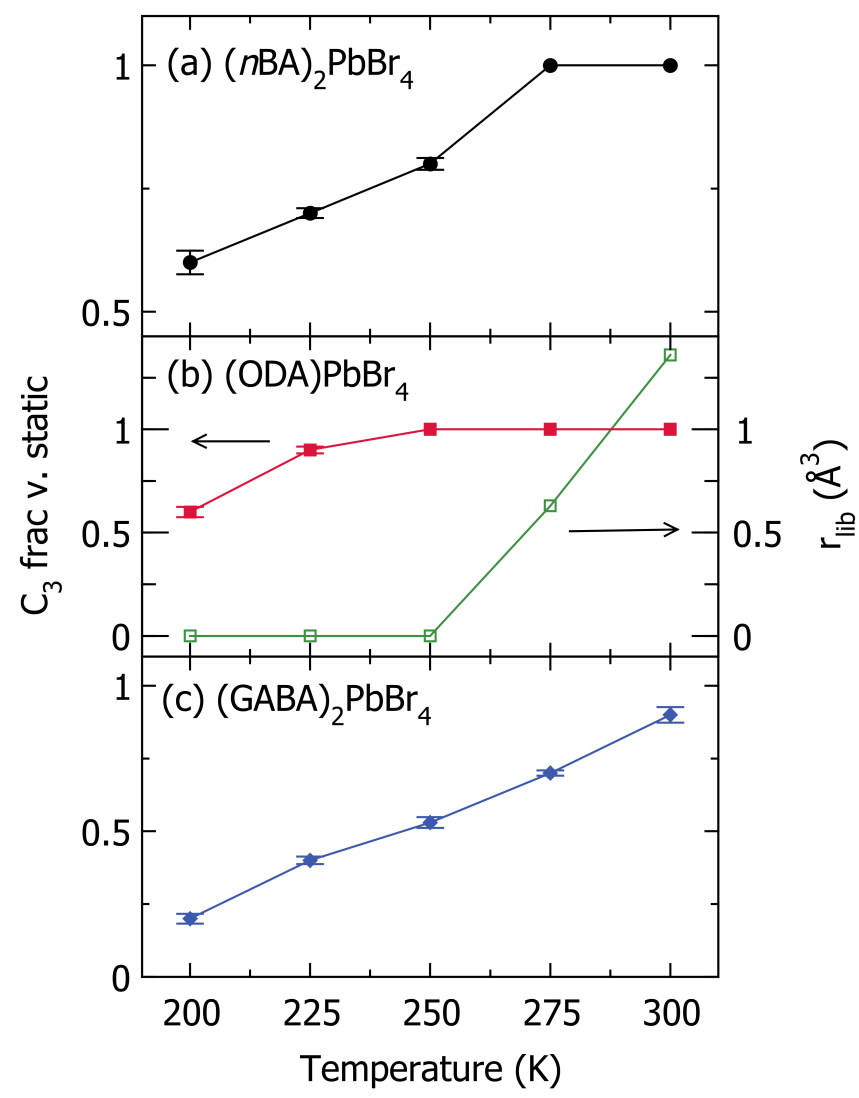

Figure S10: Fraction of $C_{3}$ motion of the organic cation in (a) $(n \mathrm{BA})_{2} \mathrm{PbBr}_{4}$ (black), (b) (ODA) $\mathrm{PbBr}_{4}$ (red), and (c) $(\mathrm{GABA})_{2} \mathrm{PbBr}_{4}$ (blue) at $200 \mathrm{~K}$ to $300 \mathrm{~K}$ extracted from QENS data at BASIS modeled by a jump model describing the $C_{3}$ rotations of the ammonium headgroup and methyl group, as described in the narrative. Values are listed in Table S2, (b, green) Libration radius of the of the $-\mathrm{CH}_{2}$ - hydrogens in (ODA) $\mathrm{PbBr}_{4}$ at $200 \mathrm{~K}$ to $300 \mathrm{~K}$ extracted from QENS data at BASIS modeled by a jump model as described in the narrative. Libration radii are as follows: At $T=300 \mathrm{~K}$ the libration distance is $1.36 \AA^{-1}$, at $T=275 \mathrm{~K}$ the libration distance is $0.630 \AA^{-1}$. 

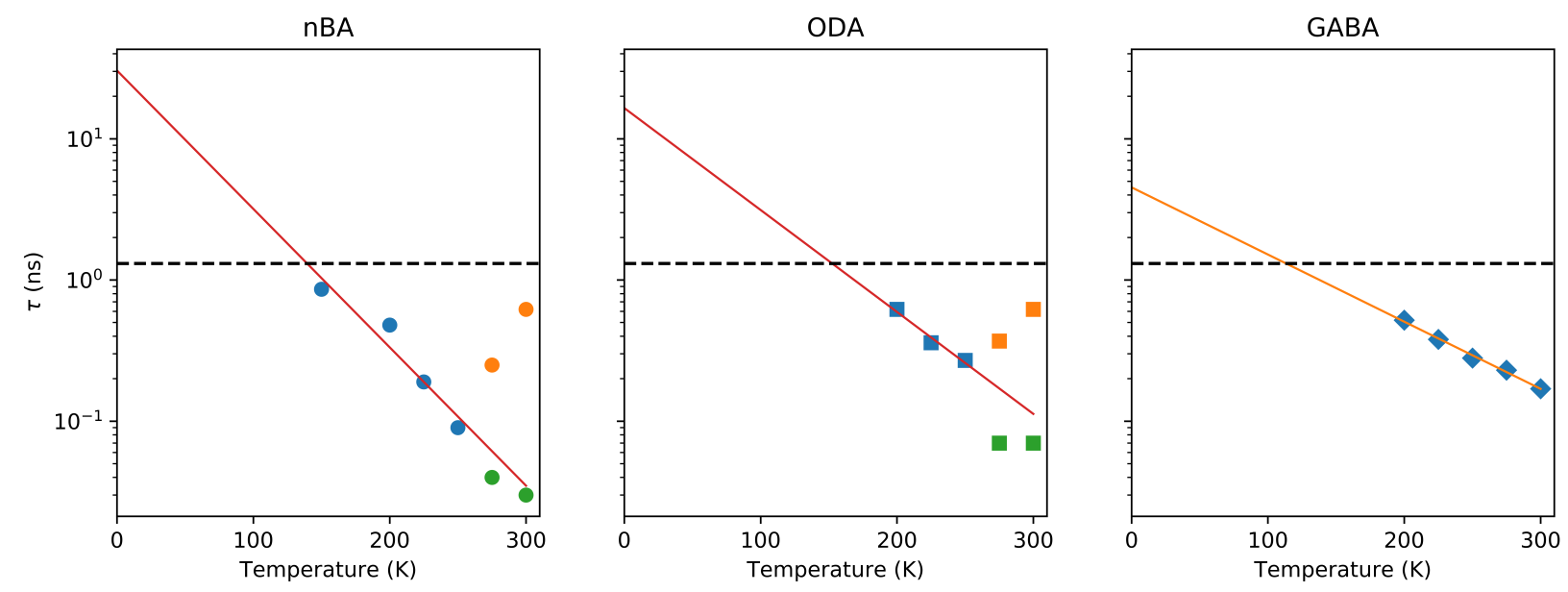

Figure S11: Extrapolated relaxation times for (left) $(n \mathrm{BA})_{2} \mathrm{PbBr}_{4}$, (middle) (ODA) $\mathrm{PbBr}_{4}$, and (right) $(\mathrm{GABA})_{2} \mathrm{PbBr}_{4}$. Extrapolations were done with a least squares polynomial fit in Python. Values determined from extrapolations are in Table $\mathbf{S 1}$. Dashed line indicates instrument resolution. Blue symbols indicate a singular Lorentzian. Orange and green symbols indicate two separate Lorentzian functions used to model the QENS data. 
Table S3: $A$-site volumes $\left(\AA^{3}\right.$, calculated from the $\left[\mathrm{NBr}_{8}\right]$ polyhedra in VESTA as described in the narrative) determined from the $295 \mathrm{~K}$ crystal structures of $(n \mathrm{BA})_{2} \mathrm{PbBr}_{4},(\mathrm{ETA})_{2} \mathrm{PbBr}_{4}$, $(\mathrm{PEA})_{2} \mathrm{PbBr}_{4}$, (MPenDA) $\mathrm{PbBr}_{4}$, (ODA) $\mathrm{PbBr}_{4},(\mathrm{BDA}) \mathrm{PbBr}_{4},(\mathrm{AEA}) \mathrm{PbBr}_{4},(\mathrm{HIS}) \mathrm{PbBr}_{4}$, and $(\mathrm{GABA})_{2} \mathrm{PbBr}_{4} \cdot 1+$

\begin{tabular}{|c|c|c|c|}
\hline Compound & Out-of-plane tilt angle $\left(^{\circ}\right)^{\mathbb{1}}$ & Volume $\left(\AA^{3}\right)$ & Observation of BBPL ${ }^{\mathbb{1}}$ \\
\hline$(n \mathrm{BA})_{2} \mathrm{PbBr}_{4}$ & 2.8 & 84.6 & No \\
$(\mathrm{ETA})_{2} \mathrm{PbBr}_{4}$ & 6.3 & 90.6 & Yes, minimal \\
$(\mathrm{PEA})_{2} \mathrm{PbBr}_{4}$ & 10.4 & 84.2 & Yes \\
$(\mathrm{MPenDA}) \mathrm{PbBr}_{4}$ & 17.9 & 78.6 & Yes \\
$(\mathrm{ODA}) \mathrm{PbBr}_{4}$ & 19.0 & 80.2 & Yes \\
$(\mathrm{BDA}) \mathrm{PbBr}_{4}$ & 20.7 & 61.1 & Yes \\
$(\mathrm{AEA}) \mathrm{PbBr}_{4}$ & 22.4 & 59.2 & Yes \\
$(\mathrm{HIS}) \mathrm{PbBr}_{4}$ & 22.8 & 79.6 & Yes \\
$(\mathrm{GABA})_{2} \mathrm{PbBr}_{4}$ & 23.9 & Yes \\
\hline
\end{tabular}

$\dagger$ Abbreviations used: $n \mathrm{BA}=n$-butylammonium, ETA $=$ ethanolammonium, PEA = phenethylammonium, MPenDA = 2-methyl-1,5-pentanediammonium, ODA =

1,8-diammoniooctammonium, BDA = butanediammonium, AEA = ammonioethylanilinium, HIS $=$ histammonium, GABA $=4$-aminobutyric acid.

\section{Time-resolved photoluminescence of $(\mathrm{GABA})_{2} \mathrm{PbBr}_{4}$}

The time-resolved photoluminescence data vary between samples. The decay times were calculated from time-resolved photoluminescence data using $R(t)=B_{1} \exp \left(-t / \tau_{1}\right)+B_{2} \exp \left(-t / \tau_{2}\right)$ and are well described by two exponential decays. The emission behavior in $(\mathrm{GABA})_{2} \mathrm{PbBr}_{4}(\mathrm{Figure}$ S12, Table [S4) is more complex; there is single-ensemble (as the spectrum does not change as a function of decay time) and multi-ensemble behavior between samples. In Figure $[\mathrm{S} 12$ at wavelengths $500 \mathrm{~nm}, 550 \mathrm{~nm}$, and $581 \mathrm{~nm}$ the behavior is primarily single-ensemble-like with spectrally sensitive time scales $\left(\approx 49 \pm 18 \mathrm{~ns}\right.$ for $\tau_{1}$ and $\approx 316 \pm 99.5 \mathrm{~ns}$ for $\left.\tau_{2}\right)$. At wavelengths $\geq 610 \mathrm{~nm}$, the decay time is much shorter $\left(\tau_{1} \approx 10 \pm 4.5 \mathrm{~ns}\right.$ and $\tau_{2} \approx 201 \pm 82 \mathrm{~ns}$ ) than in the other broad wavelengths probed indicative of multi-ensemble behavior. At $77 \mathrm{~K}$ where time-resolved data were collected for the broad spectrum the rotational motion of GABA is extrapolated to $\approx 1.5 \mathrm{~ns}$, this is faster than the decay times across all wavelengths. This varies from Figure $8 \mathrm{~b}$ in the main text; where the behavior is single-ensemble like across all wavelengths. As the rotational motions 
are not static when compared to decay times, one would expect time scales to be more spectrally independent as seen in the main text. The sample-dependent behavior is indicative of a surface or microstructure difference. ${ }^{3}$ Performing spectroscopy on single crystals would eliminate the microstructure as a variable and provide a comprehensive study on the decay lifetimes of layered perovskites. 


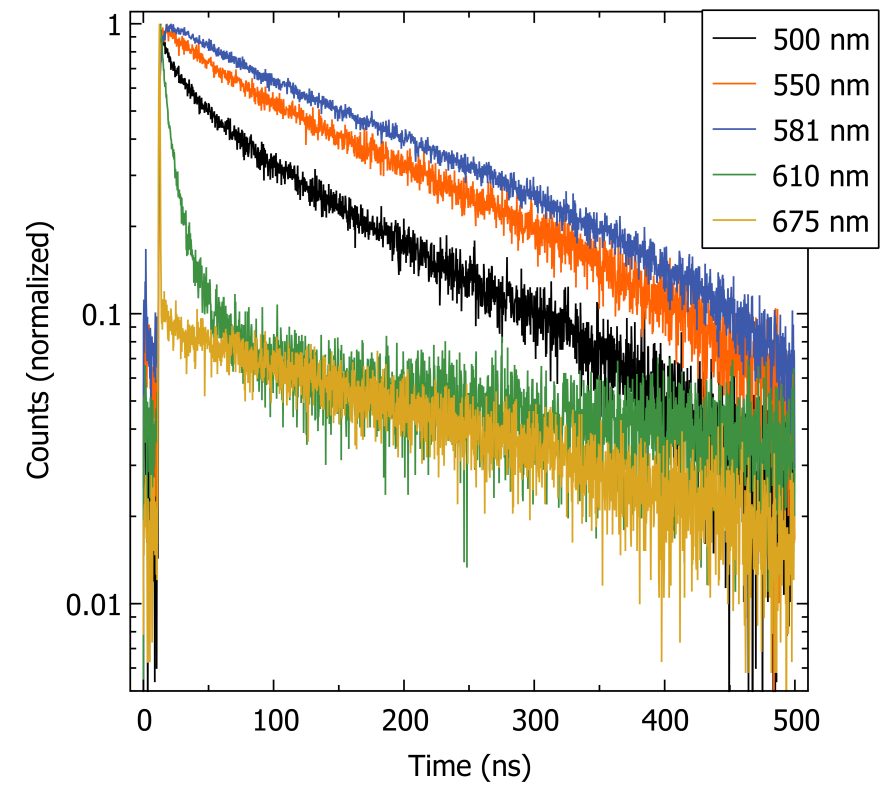

Figure S12: Time-resolved photoluminescence data for $(\mathrm{GABA})_{2} \mathrm{PbBr}_{4}$. Time-resolved photoluminescence of (GABA) ${ }_{2} \mathrm{PbBr}_{4}$ at $500 \mathrm{~nm}$ (black), $550 \mathrm{~nm}$ (red), $581 \mathrm{~nm}$ (blue), $610 \mathrm{~nm}$ (green), and $675 \mathrm{~nm}$ (yellow) at $77 \mathrm{~K}$. Data were normalized to the photoluminescence intensity at $\approx 158$ ns. Decay times are listed in Table $\mathrm{S} 4$.

Table S4: Decay times of (GABA) $)_{2} \mathrm{PbBr}_{4}$ calculated from time-resolved photoluminescence with $R(t)=B_{1} \exp \left(-t / \tau_{1}\right)+B_{2} \exp \left(-t / \tau_{2}\right)$. The data are well described by a bi-exponential decay consisting of two components. $\tau_{1}$ is shorter than $\tau_{2}$ at all wavelengths measured. Error bars represent one standard deviation.

\begin{tabular}{|c|c|c|}
\hline \multicolumn{3}{|c|}{ Decay times (ns) } \\
\hline Wavelength $(\mathrm{nm})$ & $\tau 1_{G A B A}$ & $\tau 2_{G A B A}$ \\
\hline 500 & $26 \pm 0.9$ & $190 \pm 4$ \\
550 & $51 \pm 4$ & $330 \pm 23$ \\
581 & $72 \pm 7$ & $430 \pm 47$ \\
610 & $9.5 \pm 0.2$ & $120 \pm 6$ \\
675 & $0.3 \pm 0.04$ & $283 \pm 10$ \\
\hline
\end{tabular}


EISF jump models implemented in Python: 
In [1]: import numpy as $\mathrm{np}$

from scipy.optimize import leastsq

from scipy import integrate

from scipy.optimize import curve_fit

from scipy.optimize import fsolve

In [2]: def AY(th):

\#https://en.wikipedia.org/wiki/Rotation_formalisms_in_three_dimensions

th $=$ th $*$ np.pi/180

costh $=n p \cdot \cos (t h)$

sinth $=n p \cdot \sin (t h)$

$M=n p \cdot \operatorname{array}([[\operatorname{costh}, 0, \operatorname{sinth}],[0,1,0],[-1 . * \operatorname{sinth}, 0$, costh $]])$

return $M$

def $A Z(p s):$

\#https://en.wikipedia.org/wiki/Rotation_formalisms_in_three_dimensions

ps $=$ ps $*$ np.pi/180

$\operatorname{cosps}=n p \cdot \cos (p s)$

sinps $=n p \cdot \sin (p s)$

$M=n p \cdot \operatorname{array}([[\operatorname{cosps},-1 * \operatorname{sinps}, 0],[\operatorname{sinps}, \operatorname{cosps}, 0],[0,0,1]])$

return $M$

def distance $(a, b)$ :

return np.linalg.norm(a-b) 
In [3]: \# Initial positions $(x, y, z)$ :

$\mathrm{NH} 3$ rot $=$

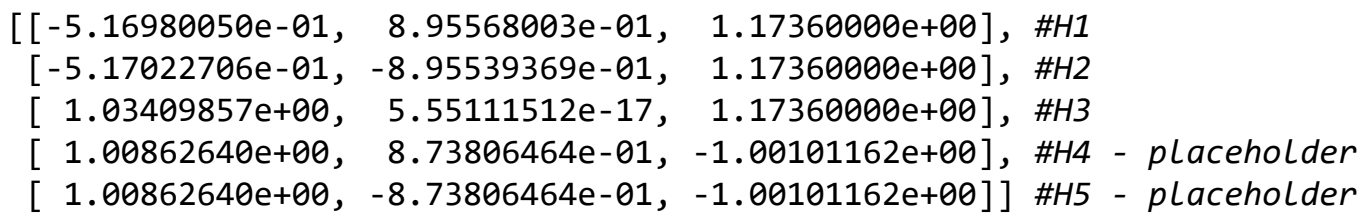

def positions(Yangle, Xoffset):

\# Generate the shifted $H$ positions based upon fitting parameters:

$\mathrm{NH} 3$ rot $2=n p \cdot \operatorname{dot}(\mathrm{AY}($ Yangle $), \mathrm{NH} 3 \operatorname{rot} . \mathrm{T}) \cdot \mathrm{T}$

$\mathrm{NH} 3$ final $=\mathrm{NH} 3$ rot2 - np.array $([$ Xoffset, $\theta, 0])$

\# Generate second set (positions 4-6) via C2 rotation:

NH3_C2 = np.dot $(A Z(180)$, NH3final. $T) \cdot T$

return NH3final, NH3_C2

def distances(Yangle, Xoffset, dlib):

NH3final, NH3_C2=positions (Yangle, Xoffset)

\# Generate jump distances for EISF calculations:

\# C2xC3 model from SI doi: 10.1063/1.5131667

\# See Table S.X.

$\mathrm{r} \theta=\operatorname{distance}(\mathrm{NH} 3 \mathrm{final}[\theta], \mathrm{NH} 3 \mathrm{final}[1]) \# \# R 1,2$

$\mathrm{r} 1$ = distance (NH3final[0], NH3_C2[0]) \#\# R1,4

$\mathrm{r} 2=$ distance (NH3final[1],NH3_C2[1]) \#\# R2,5

$\mathrm{r} 3$ = distance (NH3final[2],NH3_C2[2]) \#\# R3,6

$\mathrm{r} 4=$ distance (NH3final[0],NH3_C2[1]) \#\# R1,5

$\mathrm{r} 5=$ distance (NH3final[0],NH3_C2[2]) \#\# R1,6

$\mathrm{r} 6=$ distance $\left(\mathrm{NH} 3 \mathrm{final}[1], \mathrm{NH}_{3} \mathrm{C}_{2}[2]\right) \quad \# \# \mathrm{R} 2,6$

$\mathrm{d} 1$ = distance $(\mathrm{NH} 3$ final[4], NH3_C2[4]) \#\# R7,9

$\mathrm{d} 2=$ distance $\left(\mathrm{NH} 3 f i n a l[3], \mathrm{NH}_{-} \_\mathrm{C} 2[3]\right) \quad \# \# \mathrm{R} 8,10$

return $[r \theta, r 1, r 2, r 3, r 4, r 5, r 6, \bar{d} 1, d 2, d l i b]$

\# Calculate EISFs based upon those structures:

$j 0=$ lambda $Q, r: n p \cdot \sin \left(Q^{*} r\right) /\left(Q^{*} r\right)$

def $\operatorname{calcEISF}(Q, *$ params):

\# This is the full model, adapted from lee et al.

\# See Figure 59

Yangle $=$ params $[0]$

Xoffset $=$ params [1]

dlib=params [2]

$r 0, r 1, r 2, r 3, r 4, r 5, r 6, d 1, d 2, d l i b=$ distances (Yangle, Xoffset, dlib)

\# SI doi: 10.1063/1.5131667:

\# C2xC3, just describes the NH3 headgroup.

A_A_C2XC3 $=1 / 18 *(3+6 * j \theta(Q, r \theta)+j \theta(Q, r 1)+j \theta(Q, r 2)+j \theta(Q, r 3)+2 * j \theta(Q, r 4)+2 * j \theta(Q, r 5)$ $+2 * j \theta(Q, r 6))$

\# C2XE for CH2 adjacent to NH3 terminal group.

A_A_C $2 \times E=1 / 4 *(2+j \theta(Q, d 1)+j \theta(Q, d 2))$

\# Libration for $\mathrm{CH} 2$ groups:

A_A_C2lib $=1 / 8 *(4+4 * j \theta(Q, d l i b))$

\# Methyl rotation:

A_A_C3 $=1 / 9 *(3+6 * j \theta(Q, r \theta))$

A_total $=(3 * A$ A_C2xC3 $+2 * A$ A_C2xE $+4 * A$ A_C2lib $+3 * A$ A_C3 $) / 12$

return A_total

def calcEISF_C3lib(Q,dlib):

\# This is just $\mathrm{C} 3$ motion of the $-\mathrm{CH} 3$ and $-\mathrm{NH} 3$ ends with Libration

$\mathrm{r} \theta=$ distance $(\mathrm{NH} 3 \operatorname{rot}[\theta], \mathrm{NH} 3 \operatorname{rot}[1])$

A_A_C3 $=1 / 9 *(3+6 * j \theta(Q, r \theta))$ 


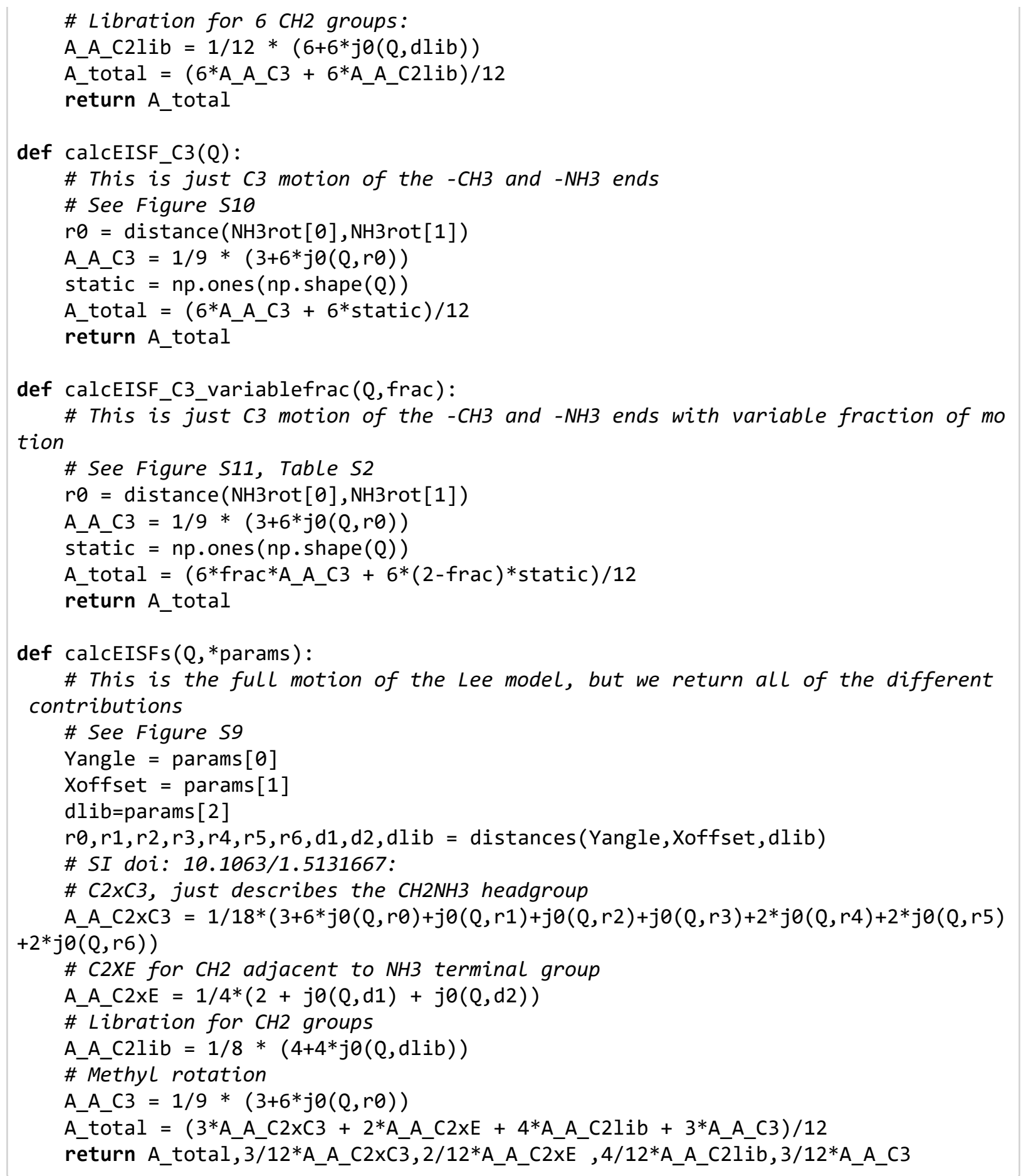




\section{References}

(1) Smith, M. D.; Jaffe, A.; Dohner, E. R.; Lindenberg, A. M.; Karunadasa, H. I. Structural origins of broadband emission from layered $\mathrm{Pb}-\mathrm{Br}$ hybrid perovskites. Chemical Science 2017, 8, 4497-4504.

(2) Hu, X.; Zhang, D.; Chen, T.; Chen, A. Z.; Holmgren, E. N.; Zhang, Q.; Pajerowski, D. M.; Yoon, M.; Xu, G.; Choi, J. J.; Lee, S.-H. Crystal structures and rotational dynamics of a twodimensional metal halide perovskite $(\mathrm{OA})_{2} \mathrm{PbI}_{4}$. The Journal of Chemical Physics 2020, 152, 014703.

(3) Thomaz, J. E.; Lindquist, K. P.; Karunadasa, H. I.; Fayer, M. D. Single Ensemble Nonexponential Photoluminescent Population Decays from a Broadband White-Light-Emitting Perovskite. Journal of the American Chemical Society 2020, 142, 1662216631. 